\title{
Tumour necrosis factor blocking agents and progression of subclinical atherosclerosis in patients with ankylosing spondylitis
}

\author{
Alper M van Sijl, ${ }^{1,2,3}$ Izhar C van Eijk, ${ }^{1}$ Mike J L Peters, ${ }^{3}$ Erik H Serné, ${ }^{3}$ \\ Irene E van der Horst-Bruinsma, ${ }^{1}$ Yvo M Smulders, ${ }^{3}$ Michael T Nurmohamed ${ }^{1,2,3}$
}

\section{Handling editor Tore K Kvien}

${ }^{1}$ Department of Rheumatology, VU University Medical Center, Amsterdam, The Netherlands ${ }^{2}$ Jan van Breemen Research Institute | Reade, Amsterdam, The Netherlands ${ }^{3}$ Department of Internal Medicine, Institute for Cardiovascular Research (ICAR), VU University Medical Center, Amsterdam,

The Netherlands

\section{Correspondence to} Alper M van Sijl, Department of Rheumatology, Jan van Breemen Research Institute Reade, Jan van Breemenstraat 2, PO Box 58271, 1040| HG, Amsterdam, The Netherlands; a.v.sij|@reade.nl

Received 12 May 2013 Revised 8 August 2013 Accepted 19 September 2013 Published Online First 3 October 2013

\section{ABSTRACT}

Background Ankylosing spondylitis (AS) is associated with an increased cardiovascular risk that might be due to the chronic underlying inflammatory process. We investigated whether subclinical atherosclerosis of the carotid artery in patients with AS was reduced after antiinflammatory treatment with tumour necrosis factor (TNF) inhibitors in a prospective observational cohort study. Methods 67 out of 81 AS patients who used TNF inhibitors and underwent ultrasonography at baseline returned for follow-up after 4.9 years. Of all patients, $12(15 \%)$ discontinued the use of TNF inhibitors. Assessments of medication use, AS-related factors and cardiovascular risk factors were measured at baseline and repeated at follow-up. B-mode carotid ultrasonography was used to investigate arterial wall parameters, including carotid intima-media thickness (cIMT) and Young's elastic modulus (YEM).

Results After a median 4.9 years of follow-up, cIMT did not change significantly (paired t test $+0.011 \mathrm{~mm}$, $p=0.561$ ) in those who continued the use of TNF inhibitors, while cIMT increased substantially $(+0.057 \mathrm{~mm}$ $p=0.069$ ) in those who did not continue their use of TNF inhibitors. The effect of TNF inhibitors was mainly mediated by a subsequent decrease in AS disease activity. Vascular elasticity (as measured with YEM) did not change significantly in patients who discontinued TNF inhibitors or those who continued TNF inhibitors. Conclusions The use of TNF inhibitors might stabilise or slow down the progression of subclinical atherosclerosis in AS patients, reflecting a decreased cardiovascular risk in these patients.

\section{INTRODUCTION}

Ankylosing spondylitis (AS), a chronic inflammatory disease of the sacroiliac joints and spine, is associated with an increased risk of cardiovascular disease. ${ }^{2}$ Although specific cardiovascular disorders (valvular disease and conduction disturbances) occur more frequently in $\mathrm{AS},{ }^{3-5}$ accelerated atherosclerosis also renders these patients more at risk of cardiovascular disease. ${ }^{6}$ Increased cardiovascular risk factors as well as the generalised inflammatory state contribute to the atherosclerotic process. ${ }^{7}$

High-resolution ultrasonography can be used to determine carotid intima-media thickness (cIMT) and vascular elasticity at the carotid arteries. In the general population, an increased cIMT is associated with an increased risk of cardiovascular disease, ${ }^{8} 9$ which was also the case in rheumatoid arthritis (RA). ${ }^{10}$
A decreased vascular elasticity indicates compromised intrinsic elastic wall properties (which can be measured with Young's elastic modulus; YEM) and is independently associated with cardiovascular disease. ${ }^{11} 12$

In AS, several studies have shown an increased cIMT and an impaired vascular elasticity. ${ }^{13-17}$ However, it is unclear whether inflammation reduction by tumour necrosis factor (TNF) inhibitors improves the risk of cardiovascular disease as measured by subclinical markers of atherosclerosis, such as cIMT and vascular elasticity. Available data on the effects of TNF inhibitors on cIMT and vascular elasticity in AS show discrepant findings, mostly due to the short follow-up period ( $<1$ year) and low number of subjects $(\mathrm{n}<30){ }^{15} \quad 18-20$ We hypothesise that TNF inhibition leads to more favourable developments in cIMT and vascular elasticity. A placebo controlled randomised trial with TNF inhibitors in patients with active AS for this particular purpose is, for ethical reasons, not possible. Therefore, we longitudinally assessed cIMT and vascular elasticity in a cohort of AS patients using TNF inhibitors at baseline, and compared those AS patients who discontinued TNF inhibitors during follow-up to those who did not.

\section{METHODS}

\section{Study population and design}

Eighty-one consecutive AS patients were included in this study at baseline from the Jan van Breemen Research Institute-Reade and VU University Medical Center in Amsterdam, The Netherlands. ${ }^{13}$ All patients fulfilled the modified New York diagnostic criteria of AS. Before inclusion in the study, all patients gave written informed consent (according to the principles reported in the Declaration of Helsinki) and the institutional ethics committee of both hospitals approved the study protocol. The present study was a prospective, observational study in patients with AS who received treatment with TNF inhibitors (70 patients on etanercept, 11 on adalimumab), when they had active disease in accordance with the Assessment of SpondyloArthritis Society (ASAS) guidelines for anti-TNF treatment. ${ }^{21}$ Clinical, laboratory and ultrasound examinations in patients were performed at baseline (before the first administration with TNF inhibitors) and after 5 years of follow-up.

\section{Ultrasound examination}

Ultrasound examination at baseline and at follow-up were performed by two observers using similar 
protocols, standard operating procedures and equipment. ${ }^{13}$ Both observers performed a reproducibility test of which the interobserver variability was good (intraclass correlation coefficient 0.861). Measurements were performed as described before. ${ }^{13}$ In short, a $7.5-\mathrm{MHz}$ linear probe, connected to a computer equipped with vessel wall movement detection software and an acquisition system (wall track system; Pie Medical) enabled measurements of the cIMT. After localisation of the right common carotid artery, cross-sectional measurements were performed $10 \mathrm{~mm}$ proximal of the carotid bulb. Sites with mural atherosclerotic plaques were avoided due to difficulty in identifying carotid arterial parameters in these regions. The distance between the lumen-intima interface and the leading edge of the media-adventitia interface of the far wall corresponds with cIMT. (Inter-adventitial) diameter was considered the distance between media-adventitia of far and near walls of the carotid artery. Change in diameter as a function of time (distension) was estimated and presented on the computer screen as distensibility. Measurements of cIMT, diameter and distension were ECG triggered to the R-peak of the cardiac cycle. Distensibility coefficient (DC) (reflecting arterial elastic properties), compliance coefficient (C) (reflecting arterial buffering capacity) and YEM (reflecting intrinsic elastic wall properties) were calculated from diameter $(\mathrm{D})$, distension $(\mathrm{d})$ and pulse pressure $(\mathrm{P}):^{13}$

$$
\begin{aligned}
& \mathrm{DC}=\left(2 \mathrm{~d} \times \mathrm{D}+\mathrm{d}^{2}\right) /\left(\mathrm{P} \times \mathrm{D}^{2}\right) \text { in } 10^{-3} \times \mathrm{kPa}^{-1} \\
& \mathrm{CC}=\pi \times\left(2 \mathrm{D} \times \mathrm{d}+\mathrm{d}^{2}\right) /(4 \times \mathrm{P}) \text { in } \mathrm{mm}^{2} \times \mathrm{kPa}^{-1} \\
& \mathrm{YEM}=\mathrm{D} /(\mathrm{cIMT} \times \mathrm{DC}) \text { in } \mathrm{kPa}
\end{aligned}
$$

\section{Other measurements}

Ascertainment of cardiovascular risk factors and AS-related factors was done at baseline and follow-up according to similar standard operating procedures. Smoking history, use of medications and history of cardiovascular risk factors and/or diseases were ascertained via questionnaire. Body mass index was calculated as the ratio of weight and squared height. Hypertension was defined as systolic blood pressure (SBP) over $140 \mathrm{~mm} \mathrm{Hg}$ and/or diastolic blood pressure (DBP) over $90 \mathrm{~mm} \mathrm{Hg}$ and/or the current use of antihypertensive medication. Mean arterial pressure was defined as $((2 \times \mathrm{DBP})+\mathrm{SBP}) / 3$. Total cholesterol, high-density lipoprotein (HDL)-cholesterol, triglycerides, erythrocyte-sedimentation rate (ESR) and C-reactive protein (CRP) were determined from fasting blood samples by enzymatic techniques, as previously described. Low-density lipoprotein (LDL)-cholesterol and the totalto HDL-cholesterol ratio were calculated. AS-related disease activity was assessed with the AS disease activity score (ASDAS) and the Bath AS disease activity index (BASDAI). ${ }^{22}$ Outcome parameters of response according to the ASAS guidelines were calculated: ASAS-20 (20\% improvement), ASAS-40 (40\% improvement) and ASAS partial remission (low disease activity). ${ }^{23}$

\section{Statistical analyses}

Results were expressed as means $\pm \mathrm{SD}$, medians (IQR) or percentages, when appropriate. Changes in cardiovascular risk factors, AS-related factors and arterial wall characteristics were analysed using paired $t$ tests, while changes in medication use were analysed using the non-parametric paired test (Wilcoxon's signed-ranks), stratified for patients who continued or discontinued the use of TNF inhibitors. To generate sufficient contrast between patients who stopped TNF inhibitors and those who continued treatment, only patients who discontinued treatment within 2.5 years $(n=9)$ were considered to have ceased TNF inhibitors. Two patients who discontinued TNF inhibitors after 2.5 years were excluded from further analyses, as these might have received sufficient beneficial effects of TNF inhibitors (due to cumulative exposure) to skew the association between the use of TNF inhibitors and changes in carotid arterial wall properties. Linear regression was used to analyse the associations between duration of use of TNF inhibitors and changes in arterial wall properties after follow-up, additionally adjusting for demographics, cardiovascular risk factors and AS-related factors at baseline. Possible confounders were considered according to available literature and by selecting variables on the basis of the effect on the original estimate of the exposure effect. Mediation of the association by changes in disease activity parameters or duration of treatment was analysed using the method of Kruskall and McKinnon. Correlations between changes in arterial wall characteristics and cardiovascular risk factors or AS-related factors were investigated using Spearman's non-parametric correlation. All data were analysed using the Statistical package for social sciences V.17.0, and results were considered statistically significant with two-sided $\mathrm{p}$ values less than 0.05 .

\section{RESULTS}

\section{Population characteristics}

In total, 81 patients were eligible for the study, of whom 69 AS patients were still on TNF inhibitors after 5 years, while nine AS patients discontinued treatment within the first 2.5 years of follow-up (median 1.0 years, range 0.2-2.3). Fourteen patients dropped out of the study because they refused follow-up visits or moved out of the area (figure 1). After approximately 5 years of follow-up, 65 AS patients, of whom 56 were still using TNF inhibitors, underwent examination. Table 1 summarises the demographic parameters at baseline of all AS patients who completed follow-up. Results were stratified according to the use of TNF inhibitors at follow-up. Compared to those who discontinued TNF inhibitors, patients who continued their use of TNF inhibitors were more often men, but were otherwise similar with respect to cardiovascular risk factors and AS-related factors (table 2). cIMT and YEM at baseline were higher in patients who later on continued their use of TNF inhibitors, albeit not statistically significantly so. Furthermore, in patients who continued their use of TNF inhibitors at 5 years of follow-up, all AS-related factors (BASDAI, ASDAS, ESR and CRP) improved significantly and more often achieved response criteria (ASAS20, ASAS40 and ASAS partial remission criteria), compared to patients who discontinued their use of TNF inhibitors.

\section{Changes in carotid arterial properties}

In patients who discontinued TNF inhibitors, cIMT increased substantially: $+0.057 \mathrm{~mm}(\mathrm{p}=0.069)$, while YEM and arterial diameter did not change $(+0.24 \mathrm{kPa}$ and $+0.2 \mathrm{~mm}$, respectively). In patients who continued their use of TNF inhibitors, cIMT remained stable $(+0.011 \mathrm{~mm}, \mathrm{p}=0.561)$, while diameter and YEM decreased $(-0.2 \mathrm{~mm}$ and $-0.29 \mathrm{kPa})$. In both group of patients, distension was reduced, while DC and CC did not change substantially (table 2). Regression analyses showed that the duration of treatment with TNF inhibitors was associated with changes in arterial wall parameters, mainly with changes in cIMT (table 3). After adjustment for the confounders age, gender, total to HDL-cholesterol ratio and ASDAS, the association between the duration of TNF inhibitor use and favourable changes in cIMT remained strong, albeit not statistically significantly, regression coefficient $(95 \% \mathrm{CI}):-0.021(-0.044$ to $0.002), p=0.073$. Additional adjustment for discontinuation of TNF inhibitors and changes in ASDAS seem to explain the association between the duration of TNF inhibitor use and favourable changes in cIMT as the regression coefficients $(95 \% \mathrm{CI})$ normalised: $-0.022(-0.070$ to 0.026$), p=0.369$ and -0.010 
Figure 1 Flowchart of study population. TNF, tumour necrosis factor.

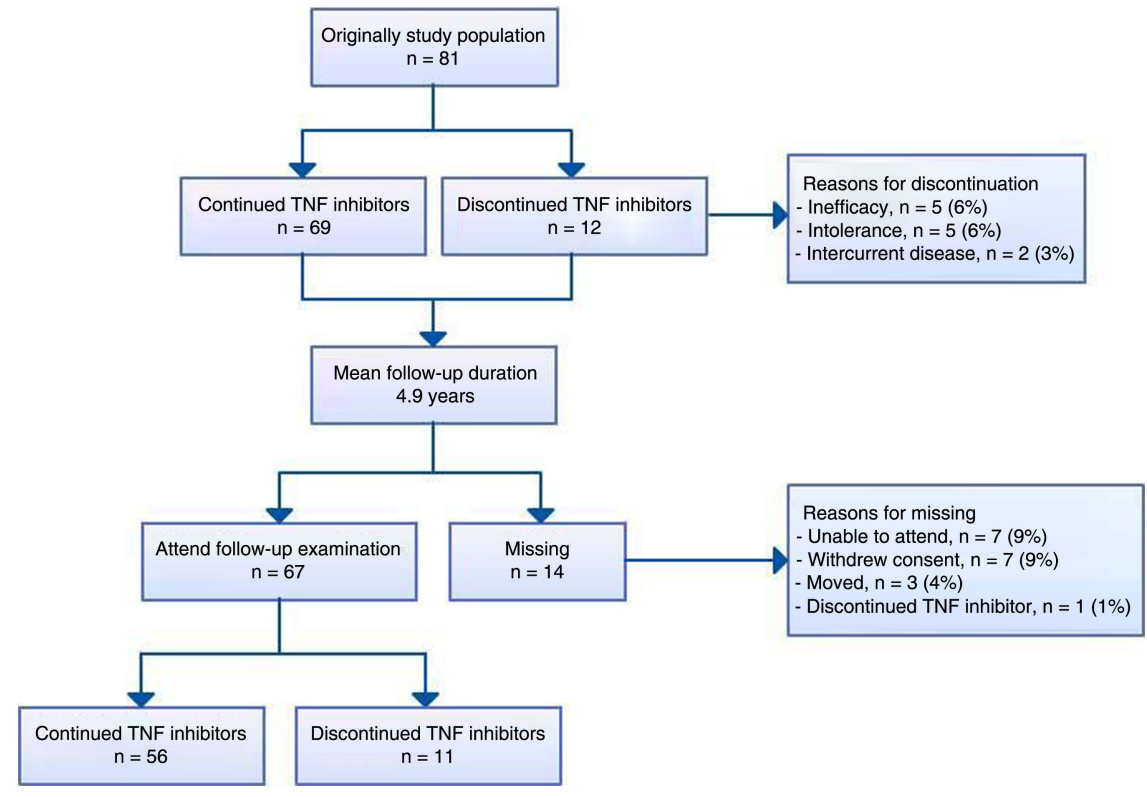

$(-0.031$ to 0.010$), p=0.319$, with a respective mediation effect of $32 \%$ and $33 \%$. No co-linearity or effect modification between duration of use of TNF inhibitors and age or disease duration was observed. In addition, although non-steroidal anti-inflammatory drug (NSAID) use decreased significantly in patients who continued to use TNF inhibitors compared to patients who discontinued TNF inhibitors (63\% vs $11 \%$ ), there was no confounding or effect modification of discontinuation of NSAID with favourable changes in arterial wall parameters (data not shown).

\section{Correlations between changes in carotid arterial parameters and cardiovascular or AS factors}

A statistically significant correlation coefficient was found between improvements in AS disease activity (BASDAI) and changes in cIMT, correlation coefficient: $0.308(\mathrm{p}=0.013)$. Results remained essentially similar when only patients who continued their use of TNF inhibitors were included in analyses.

\section{DISCUSSION}

The main results from this study indicate that in patients with AS who continue their use of TNF inhibitors cIMT progression slows down compared to normal cIMT progression in those who discontinued TNF inhibitors. In patients with AS who continued treatment with TNF inhibitors a mean increase in cIMT of $+0.011 \mathrm{~mm}$ over 5 years was observed. In comparison, earlier studies in RA have shown an annual increase in cIMT of $0.016 \mathrm{~mm} / \mathrm{year}^{24}{ }^{24}$ which is more or less comparable to the increase in cIMT over 5 years of follow-up in patients who discontinued the use of TNF inhibitors $(+0.057 \mathrm{~mm} /$ year $)$. Also, treatment with TNF inhibitors was associated with a more favourable change in cIMT, which mediation analyses have shown to be caused by the duration of treatment and changes in disease activity (as measured with BASDAI). This might imply that the continued use of TNF inhibitors or beneficial changes in AS disease activity substantially influence the association between the duration of treatment with TNF inhibitors and favourable changes in cIMT. Favourable changes in AS-related factors were correlated with favourable changes in cIMT.

To date, some studies have reported an increased cIMT and impaired vascular elasticity in AS. ${ }^{13-16} 25$ One cross-sectional study showed that AS patients on TNF inhibitors did not have a significantly higher cIMT or vascular stiffness (measured with

Table 1 Demographic factors and outcome measures

\begin{tabular}{|c|c|c|c|}
\hline & $\begin{array}{l}\text { Total population } \\
(n=65)\end{array}$ & $\begin{array}{l}\text { Discontinued TNF inhibitors } \\
\text { at follow-up }(n=9)\end{array}$ & $\begin{array}{l}\text { Continued TNF inhibitors } \\
\text { at follow-up }(n=56)\end{array}$ \\
\hline \multicolumn{4}{|l|}{ Demographic factors } \\
\hline Age, years & $44 \pm 12$ & $37 \pm 12$ & $44 \pm 12$ \\
\hline Male gender, $\%$ & 64 & 33 & $71^{*}$ \\
\hline Previous cardiovascular disease, $\%$ & 6 & 0 & 6 \\
\hline HLA-B27, \% & 73 & 67 & 75 \\
\hline Disease duration, years & $10.1(4.4-15.0)$ & $6.1(3.0-13.9)$ & $10.1(4.5-15.0)$ \\
\hline \multicolumn{4}{|l|}{ Outcome measures } \\
\hline Duration of anti-TNF treatment, years & $4.8(3.9-5.3)$ & $1.0(0.5-1.3) \dagger$ & $4.9(4.3-5.4)^{*}$ \\
\hline ASAS 20 response, $\%$ & 58 & 22 & $66^{*}$ \\
\hline ASAS 40 response, $\%$ & 49 & 22 & 55 \\
\hline ASAS partial remission, $\%$ & 21 & 0 & 25 \\
\hline
\end{tabular}


Table 2 AS and cardiovascular- related factors and arterial wall properties at baseline and follow-up

\begin{tabular}{|c|c|c|c|c|c|c|}
\hline & \multicolumn{3}{|c|}{ Discontinued TNF inhibitors at follow-up ( $n=9)$} & \multicolumn{3}{|c|}{ Continued TNF inhibitors at follow-up ( $n=56$ ) } \\
\hline & Baseline & Follow-up & Change & Baseline & Follow-up & Change \\
\hline \multicolumn{7}{|l|}{ AS-related factors } \\
\hline NSAID use, \% & 100 & 89 & -11 & 98 & 35 & $-63^{*}$ \\
\hline BASDAl, range $0-10$ & $6.7 \pm 1.2$ & $5.6 \pm 1.8$ & -1.1 & $6.1 \pm 1.3$ & $3.3 \pm 2.3$ & $-2.8^{*}$ \\
\hline $\mathrm{ESR}, \mathrm{mm} / \mathrm{h}$ & $8(3-28)$ & $9(7-18)$ & +1 & $17(6-32)$ & $5(2-18)$ & $-12^{*}$ \\
\hline $\mathrm{CRP}, \mathrm{mg} / \mathrm{L}$ & $3(1-15)$ & $4(3-10)$ & +1 & $8(3-26)$ & $3(1-6)$ & $-5^{*}$ \\
\hline ASDAS & $3.23 \pm 0.87$ & $2.87 \pm 0.89$ & -0.36 & $3.62 \pm 0.70$ & $2.04 \pm 0.99$ & $-1.6^{*}$ \\
\hline \multicolumn{7}{|l|}{ Cardiovascular risk factors } \\
\hline $\mathrm{SBP}, \mathrm{mm} \mathrm{Hg}$ & $123 \pm 22$ & $117 \pm 22$ & -6 & $125 \pm 15$ & $122 \pm 14$ & $-3^{*}$ \\
\hline $\mathrm{DBP}, \mathrm{mm} \mathrm{Hg}$ & $68 \pm 12$ & $76 \pm 12$ & +8 & $72 \pm 10$ & $80 \pm 8$ & $+8^{*}$ \\
\hline Pulse pressure, $\mathrm{mm} \mathrm{Hg}$ & $54 \pm 12$ & $41 \pm 15$ & -13 & $54 \pm 9$ & $41 \pm 10$ & -13 \\
\hline Mean arterial pressure, $\mathrm{mm} \mathrm{Hg}$ & $88 \pm 16$ & $90 \pm 14$ & +2 & $90 \pm 12$ & $94 \pm 9$ & $+4^{*}$ \\
\hline Current smoking, \% & 56 & 56 & - & 35 & 30 & -5 \\
\hline Total cholesterol, $\mathrm{mmol} / \mathrm{L}$ & $4.93 \pm 1.18$ & $5.35 \pm 2.33$ & +0.42 & $5.12 \pm 0.96$ & $5.47 \pm 1.21$ & $+0.35^{*}$ \\
\hline LDL-cholesterol, mmol/L & $2.96 \pm 0.58$ & $3.41 \pm 1.53$ & +0.45 & $3.07 \pm 0.82$ & $3.29 \pm 1.18$ & +0.22 \\
\hline HDL-cholesterol, mmol/L & $1.26 \pm 0.38$ & $1.25 \pm 0.28$ & -0.01 & $1.45 \pm 0.77$ & $1.45 \pm 0.42$ & - \\
\hline Total/HDL-cholesterol ratio & $4.09 \pm 1.02$ & $4.18 \pm 0.92$ & +0.09 & $3.89 \pm 1.09$ & $4.17 \pm 1.85$ & +0.28 \\
\hline Body-mass index, $\mathrm{kg} / \mathrm{m}^{2}$ & $29.3 \pm 9.5$ & $28.5 \pm 7.0$ & -0.8 & $26.1 \pm 4.2$ & $27.8 \pm 4.2$ & $+1.7^{*}$ \\
\hline \multicolumn{7}{|l|}{ Carotid arterial properties } \\
\hline clMT, mm & $0.626 \pm 0.077$ & $0.683 \pm 0.117$ & +0.057 & $0.655 \pm 0.107$ & $0.667 \pm 0.176$ & +0.011 \\
\hline Diameter, $\mathrm{mm}$ & $6.8 \pm 1.2$ & $7.0 \pm 1.1$ & +0.2 & $7.4 \pm 1.0$ & $7.2 \pm 1.1$ & $-0.2^{*}$ \\
\hline Distension, micrometer & $582 \pm 244$ & $451 \pm 194$ & $-131^{*}$ & $508 \pm 151$ & $444 \pm 129$ & $-64^{*}$ \\
\hline $\mathrm{DC}, \mathrm{kPa}$ & $11.1(7.6-23.4)$ & $10.2(4.7-21.3)$ & -0.9 & $9.6(5.4-15.2)$ & $10.6(6.8-16.5)$ & +1.0 \\
\hline $\mathrm{CC}, \mathrm{mm}^{2} \times \mathrm{kPa}$ & $0.5(0.3-0.6)$ & $0.5(0.2-0.7)$ & -0.01 & $0.4(0.3-0.6)$ & $0.4(0.2-0.6)$ & - \\
\hline YEM, kPa & $1.0(0.5-1.7)$ & $1.2(0.4-2.0)$ & +0.24 & $1.3(0.7-2.1)$ & $1.0(0.7-1.8)$ & -0.29 \\
\hline
\end{tabular}

pulse-wave velocity) compared to AS patients on NSAID. ${ }^{26}$ More recently, a prospective study in inflammatory arthropathies (including only a small number of AS patients) showed that after 1 year treatment with TNF inhibitors, cIMT and pulse-wave velocity improved significantly. ${ }^{19}$ Another study in 28 AS patients, treated with TNF inhibitors over 24 weeks, did not show an improvement of vascular elasticity. ${ }^{18}$

Previous results from our group already corroborated the greater cIMT in AS patients as well as an increased prevalence of myocardial infarction. ${ }^{13} 1427$ In addition, we previously demonstrated an improved endothelium-dependent vasodilation and capillary recruitment in skin (indicators of microvascular function), directly after the use of TNF inhibitors. ${ }^{15} 1728 \mathrm{In}$ line with these results, the current investigation found favourable changes in arterial wall properties with the use of TNF inhibitors that was associated with improvements in AS-related disease activity parameters and lipid levels. It is conceivable that an inflammatory-driven atherogenesis is dampened by the use of strong anti-inflammatory treatment by TNF inhibitors. Studies in RA have demonstrated that TNF inhibitors have a protective effect against cardiovascular disease incidence and several traditional and novel cardiovascular risk factors, such as endothelial

Table 3 Linear regression analyses of duration of TNF inhibitor treatment and changes in carotid arterial wall properties

\begin{tabular}{|c|c|c|c|c|c|c|}
\hline & & Changes in cIMT & Changes in distension & Changes in DC & Changes in CC & Changes in YEM \\
\hline Crude & $\begin{array}{l}\mathrm{RC}(95 \% \mathrm{Cl}) \\
\mathrm{p} \text { value }\end{array}$ & $\begin{array}{l}-0.015(-0.04 \text { to } 0.01) \\
0.156\end{array}$ & $\begin{array}{l}0.015(-0.01 \text { to } 0.03) \\
0.109\end{array}$ & $\begin{array}{l}0.30(0.71 \text { to } 1.31) \\
0.552\end{array}$ & $\begin{array}{l}0.006(-0.03 \text { to } 0.04) \\
0.711\end{array}$ & $\begin{array}{l}-0.61(-1.16 \text { to }-0.05) \\
0.033\end{array}$ \\
\hline Model 1 & $\begin{array}{l}\mathrm{RC}(95 \% \mathrm{Cl}) \\
\mathrm{p} \text { value }\end{array}$ & $\begin{array}{l}-0.017(-0.04 \text { to } 0.01) \\
0.113\end{array}$ & $\begin{array}{l}0.010(-0.01 \text { to } 0.03) \\
0.275\end{array}$ & $\begin{array}{l}0.11(-0.94 \text { to } 1.16) \\
0.833\end{array}$ & $\begin{array}{l}-0.001(-0.04 \text { to } 0.03) \\
0.958\end{array}$ & $\begin{array}{l}-0.47(-1.03 \text { to } 0.10) \\
0.104\end{array}$ \\
\hline Model 2 & $\begin{array}{l}\mathrm{RC}(95 \% \mathrm{Cl}) \\
\mathrm{p} \text { value }\end{array}$ & $\begin{array}{l}-0.020(-0.04 \text { to } 0.01) \\
0.070\end{array}$ & $\begin{array}{l}0.014(-0.01 \text { to } 0.03) \\
0.158\end{array}$ & $\begin{array}{l}0.34(-0.72 \text { to } 1.39) \\
0.525\end{array}$ & $\begin{array}{l}0.006(-0.03 \text { to } 0.04) \\
0.750\end{array}$ & $\begin{array}{l}-0.47(-1.06 \text { to } 0.11) \\
0.111\end{array}$ \\
\hline Model 3 & $\begin{array}{l}\mathrm{RC}(95 \% \mathrm{Cl}) \\
\mathrm{p} \text { value }\end{array}$ & $\begin{array}{l}-0.021(-0.04 \text { to } 0.01) \\
0.073\end{array}$ & $\begin{array}{l}0.010(-0.01 \text { to } 0.03) \\
0.324\end{array}$ & $\begin{array}{l}0.072 \text { (-1.04 to } 1.18) \\
0.896\end{array}$ & $\begin{array}{l}-0.002(-0.04 \text { to } 0.04) \\
0.914\end{array}$ & $\begin{array}{l}0.100(-0.28 \text { to } 0.48) \\
0.597\end{array}$ \\
\hline
\end{tabular}

Results expressed as regression coefficients $(95 \% \mathrm{Cl})$, with $\mathrm{p}$ values.

Model 1: Adjustment for age and gender.

Model 2: Adjustment for age, gender and total/HDL-cholesterol ratio.

Model 3: Adjustment for age, gender, total/HDL-cholesterol ratio and ASDAS.

AS, alkylosing spondylitis; ASDAS, AS disease activity score; CC, compliance coefficient; CIMT, carotid intima-media thickness; DC, distensibility coefficient; HDL, high-density lipoprotein; TNF, tumour necrosis factor; YEM, Young's elastic modulus. 
dysfunction and insulin resistance. ${ }^{29} 30$ As the progression of subclinical atherosclerosis in RA is not linear and the rate at which the cIMT increases per unit of age steepens in proportion to the RA duration, being more severe in those with disease duration longer than 10 years, ${ }^{31}$ it is possible that the beneficial effect of TNF inhibitors to decrease the progression of atherosclerosis may be more relevant in those with short disease duration before the use of this biological therapy.

Our study has some limitations that should be acknowledged. First, a single-point measuring technique was used to determine carotid arterial wall parameters. Thus, results may have been influenced by local variability of the investigated arterial segment. However, by using the same ultrasound protocol we did define the exact location on the carotid artery where measurements were made in all participants. Second, as the studied population consisted of AS patients with high disease activity, we cannot convey the same results to AS patients with low disease activity. Third, power constraints limited the comparison in changes in arterial wall parameters between patients who discontinued and continued therapy. Finally, ultrasound examination was performed at 5 years of follow-up, irrespective of the discontinuation of TNF inhibitors; therefore, the changes in cIMT that occur between discontinuation and follow-up examination are as yet unknown.

Our investigation indicates that AS patients who are treated with TNF inhibitors exhibit a substantially slower progression of cIMT compared to those who discontinued TNF inhibitors. Improvements in AS-related factors and lipids, respectively, might be related to these improvements. However, whether these favourable changes extend to a decreased cardiovascular disease incidence in AS remains to be answered. Larger studies and further investigation into the potential of TNF inhibitors protecting against cardiovascular disease in AS is warranted.

Contributors All authors contributed substantially to the conception and design, analysis and interpretation of data, drafting the article or revising it critically for important intellectual content. Final approval of the version to be published was given by all the authors.

Competing interests None.

Patient consent Obtained.

Ethics approval The institutional ethics committees of VU University Medical Center and Jan van Breemen Research Insitute-Reade approved the study protocol.

Provenance and peer review Not commissioned; externally peer reviewed.

\section{REFERENCES}

1 Peters MJ, Van der Horst-Bruinsma I, Dijkmans BA, et al. Cardiovascular risk profile of patients with spondylarthropathies, particularly ankylosing spondylitis and psoriatic arthritis. Semin Arthritis Rheum 2004;34:585-92.

2 Szabo SM, Levy AR, Rao SR, et al. Increased risk of cardiovascular and cerebrovascular diseases in individuals with ankylosing spondylitis: a population-based study. Arthritis Rheum 2011;63:3294-304.

3 Lehtinen K. Mortality and causes of death in 398 patients admitted to hospital with ankylosing spondylitis. Ann Rheum Dis 1993:52:174-6.

4 O'Neill TW, Bresnihan B. The heart in ankylosing spondylitis. Ann Rheum Dis 1992;51:705-6.

5 O'Neill TW, King G, Graham IM, et al. Echocardiographic abnormalities in ankylosing spondylitis. Ann Rheum Dis 1992;51:652-4.

6 Mathieu S, Gossec L, Dougados M, et al. Cardiovascular profile in ankylosing spondylitis. A systematic review and metaanalysis. Arthritis Care Res (Hoboken) 2010;63:557-63.

7 Bakland G, Gran JT, Nossent JC. Increased mortality in ankylosing spondylitis is related to disease activity. Ann Rheum Dis 2011;70:1921-5.
8 O'Leary DH, Polak JF. Intima-media thickness: a tool for atherosclerosis imaging and event prediction. Am J Cardiol 2002:90:18L-21L.

9 Lorenz MW, Markus HS, Bots ML, et al. Prediction of clinical cardiovascular events with carotid intima-media thickness: a systematic review and meta-analysis. Circulation 2007;115:459-67.

10 Gonzalez-Juanatey C, Llorca J, Martin J, et al. Carotid intima-media thickness predicts the development of cardiovascular events in patients with rheumatoid arthritis. Semin Arthritis Rheum 2009:38:366-71.

11 Bots ML, Dijk JM, Oren A, et al. Carotid intima-media thickness, arterial stiffness and risk of cardiovascular disease: current evidence. J Hypertens 2002;20:2317-25.

12 Mattace-Raso FU, Van der Cammen TJ, Hofman A, et al. Arterial stiffness and risk of coronary heart disease and stroke: the Rotterdam Study. Circulation 2006:113:657-63

13 Peters MJL, Van Eijk IC, Smulders YM, et al. Signs of accelerated preclinical atherosclerosis in patients with ankylosing spondylitis. J Rheumatol 2010;37:161-6.

14 Gonzalez-Juanatey C, Vazquez-Rodriguez TR, Miranda-Filloy JA, et al. The high prevalence of subclinical atherosclerosis in patients with ankylosing spondylitis without clinically evident cardiovascular disease. Medicine (Baltimore) 2009:88:358-65.

15 Mathieu S, Joly $\mathrm{H}$, Baron $\mathrm{G}$, et al. Trend towards increased arterial stiffness or intima-media thickness in ankylosing spondylitis patients without clinically evident cardiovascular disease. Rheumatology (Oxford) 2008;47:1203-7.

16 Cece $H$, Yazgan P, Karakas E, et al. Carotid intima-media thickness and paraoxonase activity in patients with ankylosing spondylitis. Clin Invest Med 2011;34:E225.

17 Hamdi W, Bouaziz MC, Zouch I, et al. Assessment of preclinical atherosclerosis in patients with ankylosing spondylitis. J Rheumatol 2012;39:322-6.

18 Capkin E, Karkucak M, Kiris A, et al. Anti-TNF-alpha therapy may not improve arterial stiffness in patients with AS: a 24-week follow-up. Rheumatology (Oxford) 2012:51:910-14.

19 Angel K, Provan SA, Fagerhol MK, et al. Effect of 1-year anti-TNF-alpha therapy on aortic stiffness, carotid atherosclerosis, and calprotectin in inflammatory arthropathies: a controlled study. Am J Hypertens 2012;25:644-50.

20 Mathieu S, Pereira B, Couderc $M$, et al. No significant changes in arterial stiffness in patients with ankylosing spondylitis after tumour necrosis factor alpha blockade treatment for 6 and 12 months. Rheumatology (Oxford) 2013;52:204-9.

21 Braun J, Davis J, Dougados $\mathrm{M}$, et al. First update of the international ASAS consensus statement for the use of anti-TNF agents in patients with ankylosing spondylitis. Ann Rheum Dis 2006:65:316-20.

22 Zochling J. Measures of symptoms and disease status in ankylosing spondylitis: Ankylosing Spondylitis Disease Activity Score (ASDAS), Ankylosing Spondylitis Quality of Life Scale (ASQoL), Bath Ankylosing Spondylitis Disease Activity Index (BASDAI), Bath Ankylosing Spondylitis Functional Index (BASFI), Bath Ankylosing Spondylitis Global Score (BAS-G), Bath Ankylosing Spondylitis Metrology Index (BASMI), Dougados Functional Index (DFI), and Health Assessment Questionnaire for the Spondylarthropathies (HAQ-S). Arthritis Care Res (Hoboken) 2011;63(Suppl. 11):S47-58.

23 Sieper J, Rudwaleit M, Baraliakos X, et al. The Assessment of SpondyloArthritis international Society (ASAS) handbook: a guide to assess spondyloarthritis. Ann Rheum Dis 2009;68(Suppl. 2):ii1-44.

24 Giles JT, Post WS, Blumenthal RS, et al. Longitudinal predictors of progression of carotid atherosclerosis in rheumatoid arthritis. Arthritis Rheum 2011:63:3216-25.

25 Bodnar N, Kerekes G, Seres I, et al. Assessment of subclinical vascular disease associated with ankylosing spondylitis. J Rheumatol 2011;38:723-9.

26 Capkin E, Kiris A, Karkucak M, et al. Investigation of effects of different treatment modalities on structural and functional vessel wall properties in patients with ankylosing spondylitis. Joint Bone Spine 2010;78:378-82.

27 Peters $M$, Visman I, Nielen $M$, et al. Ankylosing spondylitis: a risk factor for myocardial infarction? Ann Rheum Dis 2010;69:579-81.

28 Van Eijk IC, Peters MJL, Serne EH, et al. Microvascular function is impaired in ankylosing spondylitis and improves after tumour necrosis factor alpha blockade. Ann Rheum Dis 2009:68:362-6.

29 Barnabe C, Martin BJ, Ghali WA. Systematic review and meta-analysis: anti-tumor necrosis factor alpha therapy and cardiovascular events in rheumatoid arthritis. Arthritis Care Res (Hoboken) 2011:63:522-9.

30 Miranda-Filloy JA, Llorca J, Carnero-Lopez B, et al. TNF-alpha antagonist therapy improves insulin sensitivity in non-diabetic ankylosing spondylitis patients. Clin Exp Rheumatol 2012;30:850-5

31 Del Rincon I, Williams K, Stern MP, et al. Association between carotid atherosclerosis and markers of inflammation in rheumatoid arthritis patients and healthy subjects. Arthritis Rheum 2003;48:1833-40. 